isolated the enzymes, they were found to be associated with cyclin partners. CDKs regulate cell-cycle progression and are key anticancer drug targets ${ }^{6}$.

Wyllie and colleagues investigated parasites that had developed resistance to pyrazolopyrimidine compounds that was caused by exposing parasites to these molecules in the laboratory. The authors carried out wholegenome DNA sequencing to determine how this drug resistance had arisen. These results focused attention on CRK12, together with its associated cyclin protein $\mathrm{CYC} 9$, as the probable target of compound 7 . This focus was supported by their finding that parasites that express both CRK12 and CYC9 at higher than usual levels have increased resistance to the effects of compound 7. Moreover, the authors identified a mutation in the CRK12 gene in drug-resistant parasites; when this mutation was introduced into wild-type parasites, they became resistant to compound 7 (Fig. 1). The authors' computational modelling studies indicate that compound 7 binds CRK 12 in the pocket where the molecule ATP usually binds.

Although CRK12 in complex with CYC9 seems to be the primary molecular target of compound 7, it is possible that other kinases in Leishmania could be inhibited by the molecule and contribute to its anti-parasitic activity. The range of protein kinases in Leishmania is different from that in humans, so this study provides an impetus to search for other 'druggable' protein kinases in the parasite. Methods such as gene editing ${ }^{8}$ using the CRISPR-Cas9 technique have improved researchers' ability to perform large-scale genetic validation of drug targets in Leishmania. However, a major bottleneck in the drug-discovery process for neglected tropical diseases is the identification of highly specific chemical probes that allow chemical validation - evidence that confirms the molecular target of a compound of interest.

The genetic and chemical validation of CRK12 as the target of pyrazolopyrimidines is a key advance because it opens further avenues of exploration for drug discovery. If the pyrazolopyrimidines ultimately fail to be suitable for clinical use, other compounds that inhibit CRK12 could be developed. There have been only a few drug-discovery efforts targeting enzymes in Leishmania ${ }^{9}$, mainly because not many targets have been genetically or chemically validated. In this instance, a target-based approach would require the production of CRK12 in complex with CYC9, and the development of an enzyme assay that would be suitable for high-throughput screening to test libraries of chemical compounds. Protein kinases are generally amenable to such approaches, but Wyllie and colleagues report that this has proved challenging so far for CRK12.

Further research should be carried out to investigate the regulation and function of the CRK12-CYC9 complex in Leishmania to determine whether modifications such as phosphorylation regulate the activity of the complex. One key question is why is this complex essential for the survival of Leishmania in its mammalian host? The authors found that compound 7 disrupts the parasite's normal cell cycle, which is consistent with the known function of CDKs in cell division. However, the details of the molecular mechanisms at work here remain to be elucidated.

A study ${ }^{10}$ in 2016 identified the triazolopyrimidine molecule GNF6702 as having potent activity against Leishmania. It acts by inhibiting the cell's proteasomal protein-degradation machinery. Thus, in the past few years, two promising compounds with known targets have emerged. Furthermore, collaborations between pharmaceutical companies, academic institutions and the non-profit Drugs for Neglected Diseases Initiative have identified an increasing number of candidate molecules for leishmaniasis treatment that could be orally administered; these might progress from preclinical studies to clinical trials (see go.nature.com/2lc3mgn).

Is it time to consider testing such chemicals in combination with each other? Combination therapy for visceral leishmaniasis is being evaluated for current drugs because this approach increases treatment efficacy, reduces treatment duration and limits or delays the emergence of drug resistance ${ }^{11}$. The use of lower concentrations of the compounds and shorter treatment times might help to avoid the emergence of difficult-to-treat Leishmania strains, such as those that have arisen after treatment with the drug miltefosine ${ }^{12}$. Wyllie and colleagues' work might open the door for a new drug to be developed. Yet the attrition rate for drug candidates is high. More drug candidates therefore need to be identified to increase the chance that treatments for visceral leishmaniasis will make it to the clinic.

Carolina M. C. Catta-Preta and Jeremy

C. Mottram are in the Department of Biology,

University of York, York YO10 5DD, UK.

e-mail:jeremy.mottram@york.ac.uk

1. Alvar, J. et al. PLoS ONE 7, e35671 (2012).

2. Wyllie, S. et al. Nature 560, 192-197 (2018).

3. Vianna, G. Anais do $7^{0}$ Congresso Brasileiro de Medicina e Cirurgia 4, 426-428 (1912).

4. Brahmachari, U. A Treatise on Kala Azar (John Bale, Sons \& Danielsson, 1928).

5. World Health Organization. Research priorities for Chagas disease, human African trypanosomiasis and leishmaniasis (WHO, 2012).

6. Ferguson, F. M. \& Gray, N. S. Nature Rev. Drug Discov. 17, 353-357 (2018).

7. Field, M. C. et al. Nature Rev. Microbiol. 15, 217-231 (2017).

8. Beneke, T. et al. R. Soc. Open Sci. 4, 170095 (2017)

9. Jones, N. G., Catta-Preta, C. M. C., Lima, A. P. C. A. \& Mottram, J. C. ACS Infect. Dis. 4, 467-477 (2018)

10. Khare, S. et al. Nature 537, 229-233 (2016).

11.van Griensven, J. et al. Lancet Infect. Dis. 10, 184-194 (2010).

12. Rai, K. et al. mBio 4, e00611-13 (2013).

This article was published online on 25 July 2018.

\title{
ALZHEIMER'S DISEASE
}

\section{Lymphatic waste disposal in the brain}

\begin{abstract}
The discovery that a set of lymphatic vessels interacts with blood vessels to remove toxic waste products from the brain has implications for cognition, ageing and disorders such as Alzheimer's disease. SEE ARTICLE P.185
\end{abstract}

\section{MELANIE D. SWEENEY \\ \& BERISLAV V. ZLOKOVIC}

A network of lymphatic vessels acts in tandem with the blood vasculature to regulate fluid balance in the body ${ }^{1}$. The brain does not have its own lymphatic network, but the cellular membranes around the brain, known as the meninges, do have a network of lymphatic vessels. This meningeal lymphatic system was first found ${ }^{2}$ in 1787 and has been 'rediscovered' this decade ${ }^{3-5}$. Do the meningeal lymphatics have a role in brain diseases, as systemic lymphatic vessels do in systemic diseases such as cancer ${ }^{1}$ ? On page 185 , Da Mesquita et al. ${ }^{6}$ show that meningeal lymphatic vessels help to maintain both cognitive function and the proper levels of proteins in brain fluids (a process called proteostasis). The finding has implications for normal ageing and disorders such as Alzheimer's disease.

In the body, lymphatic vessels drain tissues of interstitial fluid (ISF), which contains waste products such as cellular debris and toxic molecules. The ISF forms a protein-rich fluid called lymph that circulates through the lymphatic system back to the circulating blood ${ }^{1}$. On its way, lymph is filtered through the lymph nodes, which can initiate immune responses if foreign particles are detected.

The brain does not have its own lymphatic vessels. As such, proteins and waste from the main body of the brain (the parenchyma) are transported within ISF along the walls of blood vessels to reach the cerebrospinal fluid (CSF), which circulates through the meninges ${ }^{7}$. It is well established that proteins, metabolic waste products and other molecules in these fluids can 
be removed from the brain by being transported across the walls of blood vessels, thus crossing the blood-brain barrier ${ }^{7,8}$ - a process called transvascular clearance. But it was unknown whether the meningeal lymphatic vessels are also involved in waste clearance.

Da Mesquita et al. destroyed the meningeal lymphatic vessels of mice by injecting a vesseldamaging drug into the cisterna magna - a large, CSF-filled space in the meninges. They then administered a fluorescent tracer molecule into the cisterna magna. In mice lacking meningeal lymphatic vessels, the tracer did not reach the deep cervical lymph nodes, to which the meningeal lymphatics normally drain. Similarly, injection of tracers into the brain parenchyma showed reduced ISF drainage into deep cervical lymph nodes. Previous work has shown ${ }^{9}$ that injecting high concentrations of tracer into CSF can cause the diffusion of tracer into the brain along blood vessels - but this transport was also reduced. The authors confirmed these results through several alternative approaches: using different tracers; surgically closing off drainage to the deep cervical lymph nodes; and examining mice genetically engineered so that their lymphatic-vessel development was impaired.

Destruction of the meningeal lymphatics also led to deficits in spatial orientation and fear memory. The brain's hippocampus has a key role in these behaviours, and the researchers found changes in gene expression in this region resembling those seen in neurodegenerative disorders. Collectively, these experiments suggest that drainage of brain ISF and CSF by the meningeal lymphatics is necessary for proper cognitive function.

These findings also raise an interesting question: where did the injected tracers go? One study ${ }^{10}$ has shown that tracers injected into the cisterna magna are primarily transported into the blood, and only secondarily into the lymphatic system. Simultaneous measurements of tracer movements into the meningeal lymphatics, other lymphatic vessels (for instance in the neck) and the blood might reveal whether impairment of the meningeal lymphatics leads to a shift in the pathways used to control brain proteostasis, increasing transvascular removal of waste products across the blood-brain barrier (Fig. 1), or their drainage into the venous system in the meninges ${ }^{7}$.

Da Mesquita et al. next observed an ageing-induced decrease in the diameter and coverage of meningeal lymphatic vessels, and decreased drainage of tracers from the ISF and CSF into deep cervical lymph nodes. Lymphatic-vessel growth in mice is promoted by a signalling pathway involving vascular endothelial growth factor $\mathrm{C}$ (VEGF-C) and its receptor VEGFR3, whereas impairments in the pathway lead to a loss of meningeal lymphatic vessels ${ }^{1,3}$. Furthermore, treatment with VEGF-C increases the diameter of meningeal lymphatic vessels, improving lymphatic drainage ${ }^{4}$. Consistent with

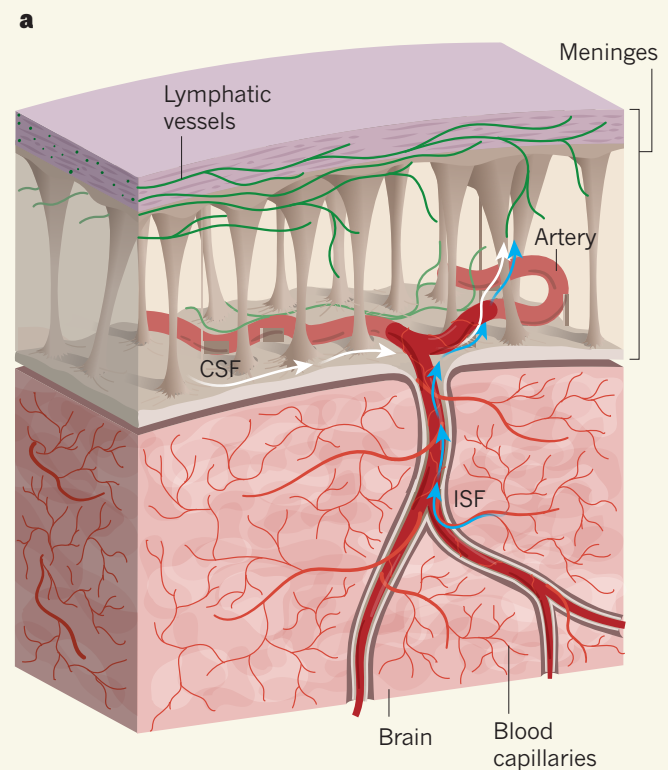

b Intact vessel system

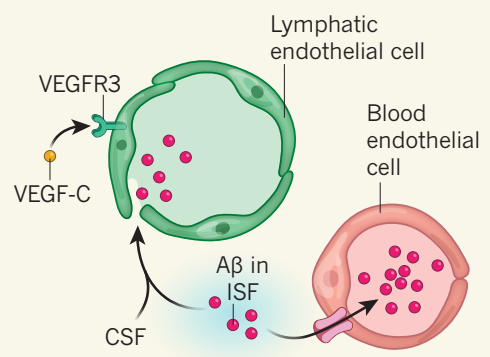

C Impaired vessel system

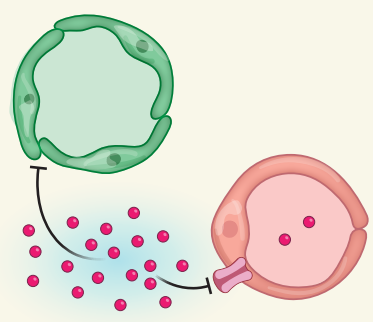

Figure 1 | Regulation of waste clearance in the brain. a, The brain does not have its own lymphatic vessels to manage the clearance of waste. Proteins and waste are transported from the brain's interstitial fluid (ISF) along blood-vessel walls to reach the cerebrospinal fluid (CSF) in a space within the meninges - membranes that cover the brain. Da Mesquita et al. ${ }^{6}$ report that lymphatic vessels in the meninges drain CSF and ISF containing waste products. b, In a healthy mouse brain, lymphatic drainage of both fluids requires signalling between vascular endothelial growth factor C (VEGF-C) and its receptor VEGFR3 on lymphatic endothelial cells lining the wall of meningeal lymphatic vessels. The protein amyloid- $\beta(A \beta)$, which is associated with Alzheimer's disease, is primarily removed from the ISF by blood vessels. c, During ageing, both vessel systems can become impaired. The diameter of the meningeal lymphatic vessels decreases, causing decreased waste clearance by this route. This defect, along with impaired clearance by blood vessels, leads to $A \beta$ accumulation in the brain.

these findings, the authors showed that local delivery of the Vegf-c gene into the cisterna magna of old mice using a virus restored the drainage of CSF tracer into deep cervical lymph nodes. This change was accompanied by restoration of spatial orientation in old mice.

Age-related impairments in transvascular clearance of waste have been implicated in the accumulation of amyloid- $\beta$ protein in the brain ${ }^{7,11,12}$ - a hallmark of Alzheimer's disease. Da Mesquita and colleagues investigated the effects of ablating the meningeal lymphatics in two mouse models of Alzheimer's disease, in which amyloid- $\beta$ protein is produced in neurons and secreted into the ISF. Ablation led to amyloid- $\beta$ accumulation in the meninges, accelerated amyloid- $\beta$ deposition in the brain parenchyma and cognitive deficits. The authors also showed that amyloid- $\beta$ had accumulated in the meninges of people who had Alzheimer's disease, pointing to the potential relevance of these findings for humans.

Notably, the researchers found that the mouse models did not develop any apparent structural or functional changes in the meningeal lymphatics at the time when amyloid- $\beta$ deposition in the brain parenchyma first became apparent. Viral delivery of Vegf-c at this time point could not prevent the cognitive impairments in either model, suggesting that the early amyloid- $\beta$ deposition and cognitive impairments in these animals were caused by disruption in another clearance pathway - most likely transvascular clearance. As transvascular-clearance routes gradually deteriorate with age, an increasing burden is probably put on the meningeal lymphatic system. If the capacity of the lymphatic system is reached, this might lead to faulty lymphatic drainage of amyloid- $\beta$ and other proteins from the ISF and CSF (Fig. 1). Thus, a dynamic relationship between the meningeal lymphatics and blood vessels seems to regulate proteostasis in the brain.

Future work should aim to improve our understanding of waste-clearance pathways from the brain, how the ISF and CSF drain into the meningeal lymphatics, and how these lymphatic vessels interact with the blood vessels at the blood-brain barrier. Such analyses will open up fresh directions for research into cognition, neurodegeneration and $\mathrm{Alz}$ heimer's disease. Da Mesquita et al. showed that strategies that promote local growth of lymphatic vessels have the potential to improve clearance by meningeal lymphatics to rebuild brain proteostasis, and might lessen amyloid- $\beta$ deposition. It remains to be determined whether treatments directed at the meningeal lymphatics can also improve the impaired function of blood vessels with age, and whether 
enhancing clearance at the blood-brain barrier can improve lymphatic drainage function.

Melanie D. Sweeney and Berislav V. Zlokovic are in the Department of Physiology and Neuroscience and at the Zilkha Neurogenetic Institute, Keck School of Medicine, University of Southern California, Los Angeles, California 90089-2821, USA. e-mail:zlokovic@usc.edu

1. Alitalo, K., Tammela, T. \& Petrova, T. V. Nature 438, 946-953 (2005).

2. Mascagni, P. Vasorum lymphaticorum corporis humani historia et ichnographia (Pazzini Carli, 1787).

3. Aspelund, A. et al. J. Exp. Med. 212, 991-999 (2015)

4. Louveau, A. et al. Nature 523, 337-341 (2015).

5. Absinta, M. et al. eLife 6, e29738 (2017).

6. Da Mesquita, S. et al. Nature 560, 185-191 (2018).
7. Sweeney, M. D., Sagare, A. P. \& Zlokovic, B. V. Nature Rev. Neurol. 14, 133-150 (2018).

8. Zhao, Z. et al. Nature Neurosci. 18, 978-987 (2015).

9. Xie, L. et al. Science 342, 373-377 (2013).

10. Courtice, F. C. \& Simmonds, W. J. Aust. J. Exp. Biol. Med. Sci. 29, 255-263 (1951)

11.Shibata, M. et al. J. Clin. Invest. 106, 1489-1499 (2000).

12.Deane, R. et al. Neuron 43, 333-344 (2004).

This article was published online on 25 July 2018.

\section{Stable and switchable polarization in 2D}

\section{The electric polarization of materials called ferroelectrics is often suppressed by an internal electric field, limiting uses for these materials. The discovery of a thin-film ferroelectric that is resistant to this field represents a major advance.}

\section{TURAN BIROL}

$\mathrm{M}$ aterials known as ferroelectrics have a macroscopic, switchable electric polarization that can be controlled by an external electric field ${ }^{1}$. This strong coupling to electric fields, however, is also the bane of ferroelectrics. Electric charges that accumulate on the surfaces of these materials produce an internal electric field called a depolarization field that, if not mitigated by external electrodes, is often large enough to suppress the polarization completely. Writing in Physical Review Letters, Xiao et al. ${ }^{2}$ report the observation of ferroelectricity that is invulnerable to the depolarization field in thin films of indium selenide $\left(\mathrm{In}_{2} \mathrm{Se}_{3}\right)$. This feature results from an atypical mechanism that drives the ferroelectricity in indium selenide, and opens the way for both the discovery of other ferroelectrics and further applications for them.

Ferroelectric polarization originates from an asymmetric distribution of atoms in a material's crystal structure - positively charged ions and negatively charged ions are slightly shifted from a symmetric distribution, in opposite directions ${ }^{3}$ (Fig. 1). However, this arrangement of atoms produces charges on the material's surface, and these charges generate a depolarization field that opposes the polarization. In thin-film ferroelectrics, if the polarization is perpendicular to the plane of the film - the preferable direction for applications - the depolarization field is

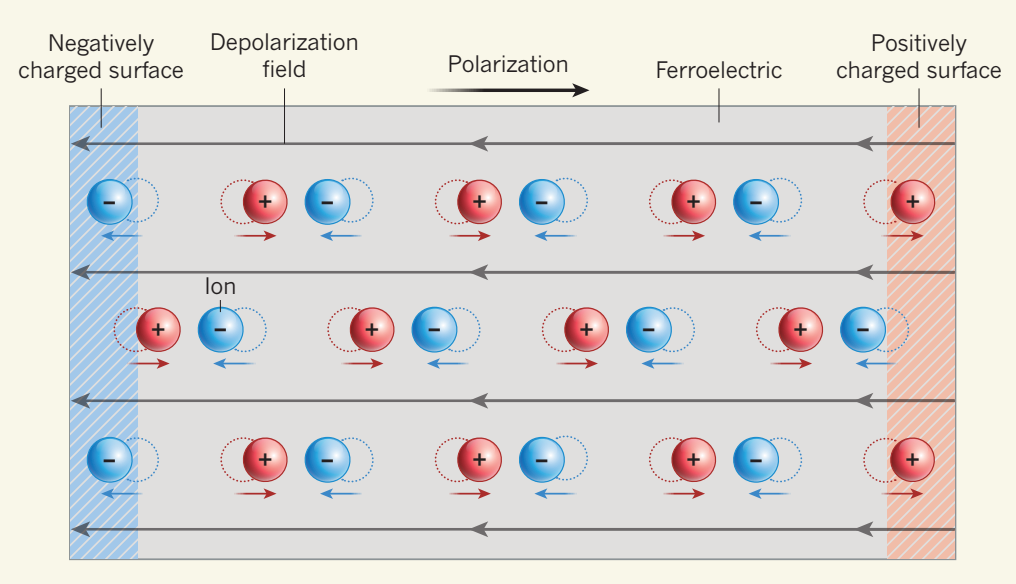

Figure 1 | Ferroelectric polarization. The electric polarization of materials called ferroelectrics originates from the fact that positively charged ions and negatively charged ions are slightly shifted from a symmetric distribution (dotted circles) in opposite directions (coloured arrows). The surfaces of ferroelectrics are negatively or positively charged owing to the presence of unpaired ions. Such charges produce an electric field known as a depolarization field that usually suppresses the polarization, limiting applications for these materials. Xiao et al. ${ }^{2}$ report a ferroelectric in which covalent bonds (not shown) between ions are sufficiently strong that the depolarization field cannot suppress the polarization. usually strong enough to suppress the polarization completely. This suppression limits the potential uses of ferroelectrics in, for example, computer memories ${ }^{4}$ and semiconductorbased electronic devices.

The most commonly studied ferroelectrics are perovskite oxides such as barium titanate $\left(\mathrm{BaTiO}_{3}\right)$. In this archetypal ferroelectric, the driving force behind the polarization is the long-range electrostatic (Coulomb) interaction between atoms. Covalent bonds, which involve the sharing of electron pairs between atoms, play a smaller part than the Coulomb interaction in determining the material's ferroelectricity ${ }^{6}$

Xiao and colleagues instead studied indium selenide, which is a chalcogenide a compound based on one of the elements in the same group of the periodic table as oxygen. Going down this group, from oxygen to sulfur to selenium, an atom's tendency to attract electrons in a chemical bond towards itself decreases. As a result, bonds have a more strongly covalent character in sulfides and selenides than in oxides, and have a larger effect on the compound's properties.

Indium selenide is a two-dimensional material that consists of five alternating indium and selenium layers, in which the indium-selenium bonds are strongly covalent. Previous theoretical work showed that there are many long-lived atomic configurations of indium selenide that differ in the local bonding environment of the ions in the material's central layers ${ }^{7}$. This work also predicted that the ferroelectric polarization in indium selenide is driven by local covalent bonds, rather than by long-range interactions, and that these bonds are strong enough to prevent the depolarization field from suppressing the polarization - even in thin films that are 3 nanometres thick (equivalent to about three sheets of indium selenide), like those of Xiao and colleagues.

Xiao et al. synthesized their films using both exfoliation (the removal of sheets from a bulk material) and a technique known as van der Waals epitaxial growth, which is an ideal method for growing materials that, like indium selenide, have weakly bound layers ${ }^{8}$. Using imaging tools such as piezoresponse force microscopy, the authors observed a polarization perpendicular to the plane of the film that is stable at temperatures of up to 700 kelvin. They also detected switching of this polarization at room temperature when an electric field was applied. 